\title{
Insights into the genetics of blood pressure in black South African individuals: the Birth to Twenty cohort
}

\author{
Liesl M. Hendry ${ }^{1,2^{*}}$, Venesa Sahibdeen ${ }^{3}$, Ananyo Choudhury ${ }^{2}$, Shane A. Norris ${ }^{4}$, Michèle Ramsay ${ }^{2,3}$,
} and Zané Lombard ${ }^{1,3}$ of the AWI-Gen study and as members of the H3Africa Consortium

\begin{abstract}
Background: Cardiovascular diseases (CVDs) are the leading cause of non-communicable disease deaths globally, with hypertension being a major risk factor contributing to CVDs. Blood pressure is a heritable trait, with relatively few genetic studies having been performed in Africans. This study aimed to identify genetic variants associated with variance in systolic (SBP) and diastolic (DBP) blood pressure in black South Africans.
\end{abstract}

Methods: Genotyping was performed using the Metabochip in a subset of participants (mixed sex; median age 17.9) and their adult female caregivers (median age 41.0) from the Birth to Twenty cohort ( $n=1947)$. Data were analysed as a merged dataset (all participants and caregivers together) in GEMMA (v0.94.1) using univariate linear mixed models, incorporating a centered relatedness matrix to account for the relatedness between individuals and with adjustments for age, sex, BMl and principal components of the genotype information.

Results: Association analysis identified regions of interest in the NOS1AP (DBP: rs112468105 $-p=7.18 \times 10^{-5}$ and SBP: rs $4657181-p=4.04 \times 10^{-5}$ ), MYRF (SBP: rs $11230796-p=2.16 \times 10^{-7}$, rs400075 $-p=2.88 \times 10^{-7}$ ) and POC1B (SBP: rs770373 $-p=7.05 \times 10^{-5}$, rs770374 $-p=9.05 \times 10^{-5}$ ) genes and some intergenic regions (DACH1 |LOC440145 (DBP: rs17240498 $-p=4.91 \times 10^{-6}$ and SBP: rs17240498 $-p=2.10 \times 10^{-5}$ ) and INTS10|LPL (SBP: rs55830938 $-p=1.30 \times 10^{-5}$, rs73599609- $p=5.78 \times 10^{-5}$, rs73667448 $\left.-p=6.86 \times 10^{-5}\right)$ ).

Conclusions: The study provided further insight into the contribution of genetic variants to blood pressure in black South Africans. Future functional and replication studies in larger samples are required to confirm the role of the identified loci in blood pressure regulation and whether or not these variants are African-specific.

Keywords: Birth to Twenty, black South Africans, blood pressure, genetics, Metabochip

\section{Background}

Cardiovascular diseases (CVDs) are the leading cause of non-communicable disease (NCD) deaths globally ahead of cancers, respiratory diseases and diabetes [1]. A major risk factor contributing to CVDs is hypertension, or raised blood pressure (BP). In 2014 the global prevalence of raised BP was approximately $22 \%$ in adults aged 18 years and older, with the highest prevalence reported in Africa at $30 \%$ for all adults combined [1]. BP and

\footnotetext{
*Correspondence: liesImaryhendry@gmail.com

'School of Molecular \& Cell Biology, Faculty of Science, University of the Witwatersrand, Johannesburg, South Africa

${ }^{2}$ Sydney Brenner Institute for Molecular Bioscience, Faculty of Health Sciences, University of the Witwatersrand, Johannesburg, South Africa Full list of author information is available at the end of the article
}

hypertension are heritable, multifactorial traits, with a significant genetic contribution (approximately 30-50\% heritable [2]) in addition to various non-genetic risk factors. The genetic contribution is polygenic, with small contributions from risk alleles in multiple genes playing a role in the aetiology of the trait or disorder [3]. Numerous studies have already reported on genes and variants that have associations with hypertension, systolic blood pressure (SBP) and diastolic blood pressure (DBP). To date, however, most of the large-scale studies have been in individuals of European ancestry [4], with fewer studies conducted in individuals of Asian or African ancestry [5]. African-related research has also largely been carried out in African-Americans with 
studies conducted in cohorts from the African continent being limited.

The current study aims to elucidate the role of genetic polymorphisms in blood pressure variance in a black South African population.

\section{Methods}

\section{Study participants}

This study included all available DNA samples from African ancestry Birth to Twenty (Bt20) participants (mean age of 17.9 years with no minors at time of phenotype data collection) and their female caregivers (mean age of 41.9 years at time of phenotype data collection). The Bt20 cohort forms the basis of the largest longitudinal study on child and adolescent health and development in Africa. The cohort initially enrolled babies born as single births to women residing in Soweto, Johannesburg during a 7-week enrolment period between April and June 1990 and phenotype data has been collected at various time points since then [6].

Written assent was obtained from all participants at 13 years of age in conjunction with written consent from caregivers prior to blood sample collection. Written consent was obtained from participants when they were over 18 years of age. Ethical clearance was obtained from the University of the Witwatersrand Human Research Ethics Committee (Medical) for collection of DNA samples and phenotype data from this cohort (M010556). Further clearance was obtained for use of these samples to identify genetic risks associated with blood pressure (M1411116) in a black South African population. DNA is currently stored in the Division of Human Genetics at the National Health Laboratory Service (NHLS), Braamfontein, South Africa.

\section{Phenotype measurements}

$\mathrm{BP}$ readings were taken and weight and height measured as described previously [7]. BP readings were taken with participants in a seated position. After five minutes of sitting in a resting position, three measurements were taken at intervals of two minutes. The first reading was discarded, in case of possible "white coat syndrome", and an average of the second and third measurements was calculated and used in all analyses. Body mass index (BMI) was calculated as weight $(\mathrm{kg})$ divided by height squared $\left(\mathrm{m}^{2}\right)$. The phenotype data used in this study was from the year 13 and 17/18 data collection time points for the female caregivers and participants, respectively.

\section{Genotyping}

DNA, extracted from blood using the salting out method [8], was normalized to $50 \mathrm{ng} \cdot \mathrm{ul}^{-1}$ prior to genotyping at the UC Davis Genome Centre (California, USA) using the Metabochip (Illumina, San Diego, CA, USA). The
Metabochip is a custom genotyping array that allows for the genotyping of almost 200,000 single nucleotide polymorphisms (SNPs) known to influence cardiometabolic traits [9]. The DNA samples were genotyped in two separate batches (participants and caregivers) and duplicate samples from each batch (nine in total) were sent with the unique samples to assess genotyping consistency. Genotypes were called using GenomeStudio Software for Illumina (v2011.1) and a custom DNAtech cluster file and final output was provided as final reports in the forward strand orientation.

\section{Data quality control}

Pre-analysis quality control (QC) of the data was carried out separately for the two datasets using PLINK (v1.9) $[10,11]$, SMARTPCA (to run the principal component analysis (PCA) for identification of population outliers [12]) and Genesis (to visualize the PCA) [13]. Final report files were converted into binary PLINK format files. An initial SNP and sample removal step involved removing SNPs with complete missing genotype data and poorly genotyped samples (more than 20\% missing genotype data). Further SNP QC involved removal of SNPs with high missingness rate $(>2 \%)$, low minor allele frequency (MAF) $(<0.01)$ and those failing HardyWeinberg equilibrium (HWE) $\left(p<1 \times 10^{-5}\right)$. Additional sample QC involved removal of samples with high missingness rate $(>2 \%$ for caregivers and $>3 \%$ for participants), those with discordant sex, related samples (PI_HAT >0.1875), duplicates, samples with extreme heterozygosity (heterozygosity rate \pm 3 standard deviations from the mean [14]) and population outliers (identified by manual inspection as those individuals falling significantly out of the main cluster in the PCA plots). One SNP (out of all those associated with DBP and SBP) was also disregarded due to poor clustering examined in the cluster plots generated in Evoker [15]. Checks were also carried out on the phenotype data and corrections were made where inconsistencies were found between the original questionnaires and captured data.

\section{Association analysis}

Data were analysed as a merged dataset (all participants and caregivers together). Merging of the datasets in PLINK resulted in 1947 individuals and 125,906 SNPs remaining for analysis. Analysis was performed in GEMMA (v0.94.1) [16] using univariate linear mixed models and incorporating a centered relatedness matrix (calculated by GEMMA using the given genotypes) to account for the relatedness between individuals. BP was analysed as a continuous variable and included adjustments for age, sex and BMI. Principal components (PCs) were also included if necessary after examination of quantile-quantile (Q-Q) plots, which were constructed 
using R (v3.0.3) [17]. The number of PCs to include was determined by an improvement seen in the Q-Q plots after re-analysis with various numbers of PCs included: no PCs were deemed necessary to include for the analysis with DBP, while the first 10 PCs were included for the analysis with SBP. A Bonferroni array-wide significance threshold to correct for multiple testing was calculated as $0.05 /$ number of independent markers: $p<$ $6.7 \times 10^{-7}(0.05 / 74475)$. The number of independent markers was calculated by performing linkage disequilibrium (LD) based SNP pruning where a window of 50 SNPs was considered at a time, LD between each pair of SNPs in the window was calculated and one of a pair of SNPs was removed if the LD was greater than 0.5. To address the possible introduction of type II errors through the application of this rigorous correction, we chose to also present results where a cut-off of $p \leq 1 \times 10^{-4}$ was met, as results that may provide interesting biological leads.

\section{Results}

This association analysis focused on 1947 African individuals (participants and their caregivers) from the Bt20 cohort. Descriptive statistics of the dataset are shown in Table 1.

All SNPs associated with DBP or SBP at $\leq 1 \times 10^{-4}$ are presented in Table 2. Interestingly, SNPs in introns in NOS1AP and intergenic to DACH1|LOC440145 were found to be associated with both DBP and SBP. In addition, two SNPs intronic to MYRF and POC1B and three SNPs intergenic to INTS10|LPL associated with SBP. The only SNPs that reached array-wide significance are the two intronic SNPs in MYRF (rs11230796-G and rs400075-T) associated with SBP. Genome-wide visualisations of the associations, with these regions highlighted, are shown in the Manhattan plots in Fig. 1. Regional plots centered around the lead SNPs of NOS1AP (DBP and SBP), MYRF (SBP), POC1B (SBP) and the intergenic region of INTS10|LPL (SBP), and plotted against European (CEU) and Yoruban (YRI) LD backgrounds, are also shown (Figs. 2, 3, 4, 5 and 6).

Table 1 Descriptive statistics of the individuals remaining after QC in the merged dataset

\begin{tabular}{cllll}
\hline & & Mean (SD) & Median & Range \\
\hline Merged $(n=1947)$ & & & & \\
Females: 73.3\% & Age (years) & $30.1(13.5)$ & 30.0 & $17.3-84.0$ \\
Males: 26.6\% & Weight (kg) & $68.0(16.7)$ & 64.4 & $36.1-136.6$ \\
& Height (m) & $1.6(0.1)$ & 1.6 & $1.2-1.9$ \\
& BMI (kg.m $\left.{ }^{-2}\right)$ & $26.2(7.1)$ & 24.6 & $14.9-58.8$ \\
& SBP (mmHg) & $117.8(16.8)$ & 116.0 & $77.0-206.5$ \\
& DBP (mmHg) & $74.1(11.1)$ & 72.5 & $44.5-129.5$ \\
\hline
\end{tabular}

\section{Discussion}

Much of the research focus in Africa has centred on infectious diseases, due to its high burden in this part of the world. NCDs are, however, gaining increasing interest and becoming as significant due to their increasing role in morbidity and mortality on the continent. This study aimed to investigate the genetics of BP in black South Africans and revealed several associations with DBP and SBP in black South Africans. The analysis pointed to regions of interest in the NOS1AP (DBP and SBP), MYRF (SBP) and POC1B (SBP) genes as well as two intergenic regions (DACH1|LOC440145 and INTS10|LPL). Two SNPs in the MYRF gene met the array-wide significance threshold.

The gene with the most plausible functional link to BP regulation or hypertension risk in this study is the NOS1AP (nitric oxide synthase 1 (neuronal) adaptor protein) gene. In our study, two SNPs in this gene, rs112468105-G and rs4657181-T, associated with increased DBP and decreased $\mathrm{SBP}$, respectively. Polymorphisms in this gene have previously been associated with other cardiovascular phenotypes, most notably QT interval length [18]. Interestingly, several genes in the chromosome 1q linkage region, in which NOS1AP falls, have previously been reported to be associated with hypertension [19] which motivates for this gene and regions on chromosome 1 to be investigated further for their role in $\mathrm{BP} /$ hypertension. The gene encodes an adaptor protein for neuronal nitric oxide synthase (nNOS), an enzyme involved in nitric oxide (NO) synthesis in the nervous tissue in the central and peripheral nervous systems. NO has several roles in the body, one of which is as a vasodilator and regulator of vascular tone and blood flow [20]. NO has also been implicated in BP regulation and impaired NO bioactivity has shown to be associated with hypertension, although the mechanism is unclear [21]. Research has also shown that NO synthesised in the central nervous system by nNOS is involved in the central regulation of blood pressure and inhibition of nNOS activity in the medulla and hypothalamus has been linked to systemic hypertension [22]. The exact role of the NOS1AP protein in the regulation of nNOS function in human disease is not clear, but a recent study showed that over-expression of NOS1AP increased nNOS activity [23].

Despite associations of the two SNPs in MYRF (myelin regulatory factor) (rs11230796-G and rs400075-T) with increased SBP at array-wide significance $\left(p<6.7 \times 10^{-7}\right)$, there is no clear functional link to BP. Polymorphisms in this gene have not shown any previous associations with BP or hypertension, but have been associated with fatty acid, phospholipid and blood metabolite levels [24-27].

There is some correlation of our findings with BP and other cardiometabolic-related trait associations found by studying European, Asian and African-American cohorts (Additional file 1: Table S1). Input of our top associated SNPs into the NHLBI GRASP catalog, v2.0.0.0 [28] and 
Table 2 All SNPs associated with DBP or SBP at $p \leq 1 \times 10^{-4}$

\begin{tabular}{|c|c|c|c|c|c|c|c|c|c|c|c|}
\hline \multirow[t]{2}{*}{ Phenotype } & \multirow[t]{2}{*}{ Chromosome } & \multirow[t]{2}{*}{ Gene/Region } & \multirow[t]{2}{*}{ SNPID ${ }^{a}$} & \multirow[t]{2}{*}{ Base pair position $^{\text {a }}$} & \multicolumn{2}{|c|}{ Alleles } & \multicolumn{3}{|c|}{ A1 Frequency ${ }^{c}$} & \multirow[t]{2}{*}{$P$-value ${ }^{d}$} & \multirow[t]{2}{*}{ Beta } \\
\hline & & & & & $\overline{\mathrm{A} 1^{\mathrm{b}}}$ & $\overline{\mathrm{A} 2}$ & Bt20 & YRI & $\overline{\text { CEU }}$ & & \\
\hline \multirow[t]{10}{*}{ DBP } & 13 & DACH1 | LOC440145 & rs17240498 & $72,965,307$ & C & $\mathrm{T}$ & 0.01 & 0.00 & 0.18 & $4.91 \times 10^{-6}$ & 7.93 \\
\hline & 21 & ADAMTS5 | C210rf94 & rs469709 & $28,801,007$ & A & G & 0.05 & 0.02 & 0.13 & $9.21 \times 10^{-6}$ & 3.50 \\
\hline & 10 & LOC642666 | LOC727960 & rs12761063 & $82,533,425$ & $\mathrm{~T}$ & C & 0.11 & 0.12 & 0.10 & $1.58 \times 10^{-5}$ & 2.47 \\
\hline & 2 & PLEKHH2 | LOC728819 & rs13423605 & $43,886,460$ & C & $\mathrm{T}$ & 0.13 & 0.18 & 0.04 & $2.25 \times 10^{-5}$ & 2.29 \\
\hline & 12 & SCARB1 & rs10846744 & $125,312,425$ & G & $C$ & 0.23 & 0.24 & 0.85 & $2.49 \times 10^{-5}$ & -1.79 \\
\hline & 2 & ARL6IP6 | LOC391453 & rs 2114653 & $153,648,587$ & G & A & 0.16 & 0.14 & 0.36 & $4.28 \times 10^{-5}$ & -1.94 \\
\hline & 19 & $E M L 2 \mid G I P R$ & rs4994276 & $46,164,172$ & $\mathrm{~T}$ & C & 0.15 & 0.20 & 0.19 & $5.87 \times 10^{-5}$ & -1.96 \\
\hline & 12 & TRPV4 & rs16939725 & $110,250,587$ & G & A & 0.14 & 0.14 & 0.02 & $7.08 \times 10^{-5}$ & -2.04 \\
\hline & 1 & NOSIAP & rs112468105 & $162,195,649$ & G & C & 0.01 & 0.02 & 0.00 & $7.18 \times 10^{-5}$ & 6.69 \\
\hline & 12 & DNAHIO & rs6488908 & $124,377,814$ & G & A & 0.13 & 0.14 & 0.04 & $7.94 \times 10^{-5}$ & -2.02 \\
\hline \multirow[t]{17}{*}{ SBP } & 11 & MYRF & rs11230796 & $61,529,267$ & G & $\mathrm{T}$ & 0.06 & 0.06 & 0.22 & $2.16 \times 10^{-7}$ & 6.12 \\
\hline & 11 & MYRF & rs400075 & $61,528,814$ & T & C & 0.06 & 0.06 & 0.22 & $2.88 \times 10^{-7}$ & 6.02 \\
\hline & 8 & INTS10 | LPL & rs55830938 & $19,735,188$ & G & $\mathrm{T}$ & 0.03 & 0.03 & 0.00 & $1.30 \times 10^{-5}$ & 7.49 \\
\hline & 10 & LOC100128511|C10orf114 & rs6482175 & $21,573,536$ & C & $\mathrm{T}$ & 0.08 & 0.11 & 0.19 & $1.42 \times 10^{-5}$ & 4.31 \\
\hline & 13 & DACH1 | LOC440145 & rs17240498 & $72,965,307$ & C & $\mathrm{T}$ & 0.01 & 0.00 & 0.18 & $2.10 \times 10^{-5}$ & 11.68 \\
\hline & 1 & FMO4 | BAT2D1 & rs10798391 & $171,389,938$ & $\mathrm{~T}$ & G & 0.02 & 0.01 & 0.19 & $2.19 \times 10^{-5}$ & 8.16 \\
\hline & 1 & NOSIAP & rs4657181 & $162,255,385$ & T & A & 0.05 & 0.02 & 0.56 & $4.04 \times 10^{-5}$ & -5.62 \\
\hline & 15 & CYP19A1 & rs10459592 & $51,536,141$ & G & T & 0.20 & 0.27 & 0.58 & $5.75 \times 10^{-5}$ & 2.81 \\
\hline & 8 & INTS10 | LPL & rs73599609 & $19,756,974$ & C & G & 0.05 & 0.05 & 0.00 & $5.78 \times 10^{-5}$ & 5.14 \\
\hline & 8 & INTS10 | LPL & rs73667448 & $19,747,475$ & C & A & 0.03 & 0.04 & 0.00 & $6.86 \times 10^{-5}$ & 6.72 \\
\hline & 12 & POC1B & rs770373 & $89,818,289$ & T & C & 0.18 & 0.33 & 0.45 & $7.05 \times 10^{-5}$ & -2.95 \\
\hline & 11 & STK33 & rs1596888 & $8,455,344$ & T & G & 0.43 & 0.48 & 0.72 & $7.51 \times 10^{-5}$ & 2.20 \\
\hline & 3 & C3orf17|BOC & rs1881941 & $112,852,476$ & A & T & 0.12 & 0.72 & 0.93 & $7.66 \times 10^{-5}$ & 3.39 \\
\hline & 22 & FLJ46257| FAM19A5 & rs6519991 & $48,725,535$ & A & G & 0.17 & 0.16 & 0.10 & $8.31 \times 10^{-5}$ & -2.89 \\
\hline & 7 & TAXIBP1 & rs6944913 & $27,826,523$ & G & A & 0.02 & $<0.01$ & 0.24 & $8.63 \times 10^{-5}$ & 8.09 \\
\hline & 11 & KCNQ1 & rs1557647 & $2,551,363$ & $A$ & G & 0.26 & 0.32 & 0.68 & $9.00 \times 10^{-5}$ & 2.51 \\
\hline & 12 & $P O C 1 B$ & rs770374 & $89,818,022$ & $\mathrm{~T}$ & G & 0.23 & 0.37 & 0.56 & $9.05 \times 10^{-5}$ & -2.62 \\
\hline
\end{tabular}

${ }^{a}$ All SNP IDs and base pair positions are reported using GRCh37 (Genome Reference Consortium human genome Build 37)

${ }^{\mathrm{b}} \mathrm{A} 1$ corresponds to the minor allele in the dataset

'Frequencies of allele 1 are recorded for the dataset used in this study (Bt20) and for an African and European 1000 Genomes population - the Yoruba in Ibadan, Nigeria (YRI) and the Utah Residents with Northern and Western Ancestry (CEU)

${ }^{\mathrm{d}} p$-value adjusted for age, sex, BMI and principal components. $P$-values that pass the "array-wide" significance threshold $\left(p<6.7 \times 10^{-7}\right)$ are shown in bold

'Beta values are with respect to the minor allele in the sample. A positive beta indicates that the minor allele is associated with an increased blood pressure

relative to the major allele, and vice versa

PhenoScanner [29] revealed one SNP (rs10798391) with a previous marginal association with SBP and DBP in European individuals [30]. rs10846744, associated with DBP in this study, showed previous associations with lipoprotein associated phospholipase A2 activity [31, 32] and rs11230796, associated with SBP at the array-wide significance level in this study, previously associated with serum linoleic acid and other polyunsaturated fatty acid levels in European individuals at a genome-wide significance level [33]. In addition, associations have been recorded between SNPs in ARL6IP6 and SBP or DBP in African and Asian individuals [34, 35]; MYRF and DBP in
Europeans [30]; KCNQ1 and DBP, SBP or early onset hypertension in African, European or mixed populations [30, 36-38]; NOS1AP, PLEKHH2 and POC1B and SBP or DBP in Europeans [30]; SCARB1 and SBP in Europeans [30]; and STK33 and TAX1BP1 and hypertension in Europeans [39]. However, none of these associations were at a genome-wide significance level. Of importance to note is that our findings do not overlap with associations at a genome-wide significance level in large scale studies conducted in African Americans, including a recent study by Liang and colleagues [40]. 

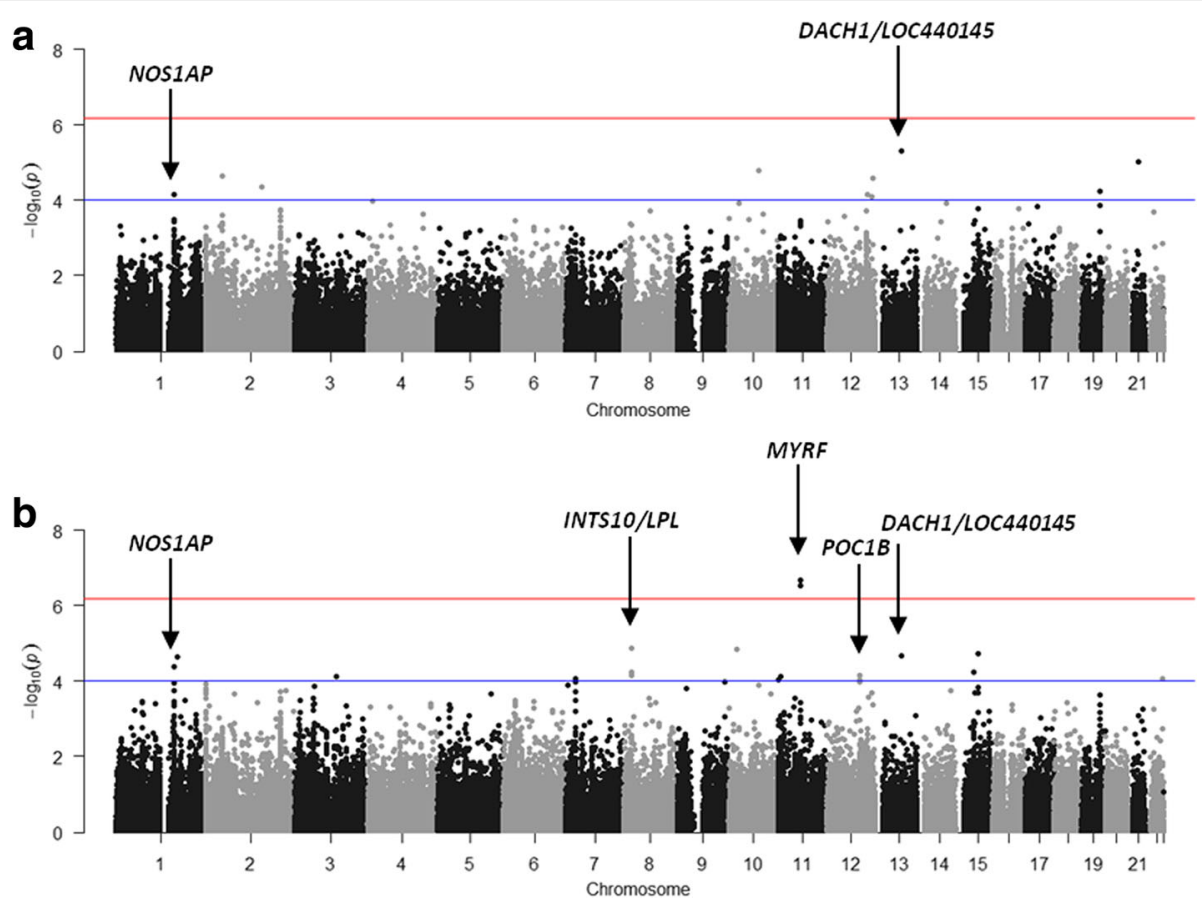

Fig. 1 Manhattan plots drawn from the association results (with correction for covariates and PCs where necessary). Plots are shown for association with (a) DBP and (b) SBP. The upper horizontal line indicates the calculated array-wide significance cutoff $\left(p=6.7 \times 10^{-7}\right)$ while the lower horizontal line shows the cutoff of $p=1 \times 10^{-4}$. Identified regions of interest for further investigation are indicated by arrows. DACH1 - Dachshund Family Transcription Factor 1; INTS10 - integrator complex subunit 10; LPL - lipoprotein lipase; MYRF - myelin regulatory factor; NOS1AP - nitric oxide synthase 1 (neuronal) adaptor protein; $P O C 1 B$ - POC1 centriolar protein

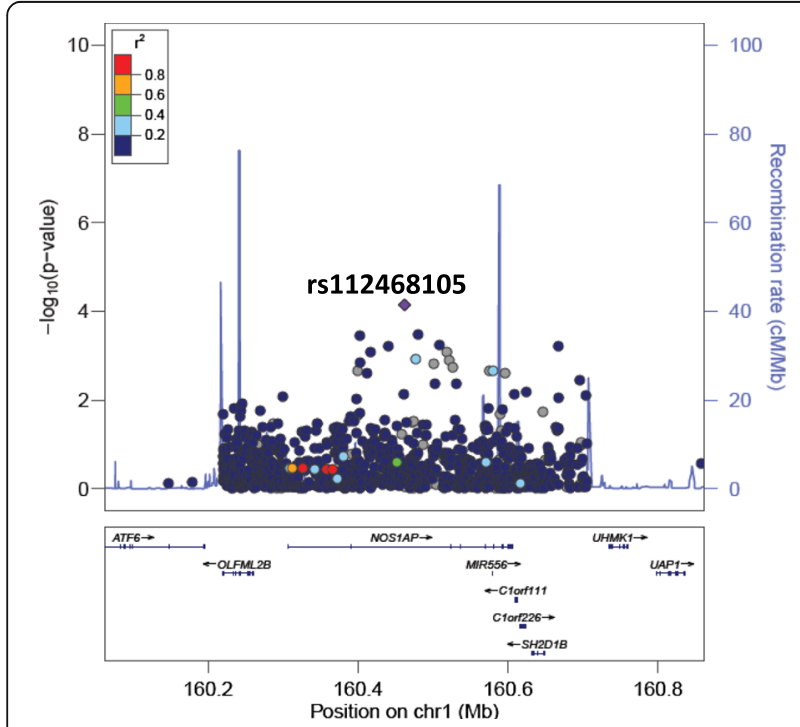

Fig. 2 LocusZoom plot for the association of rs112468105 (in NOS1AP) with DBP against a YRI LD background. rs112468105 is represented by a purple diamond. SNPs around this index SNP are coloured according to the LD between each SNP and the index SNP. SNPs with missing LD information are shown in grey. rs112468105 is monomorphic in the CEU population

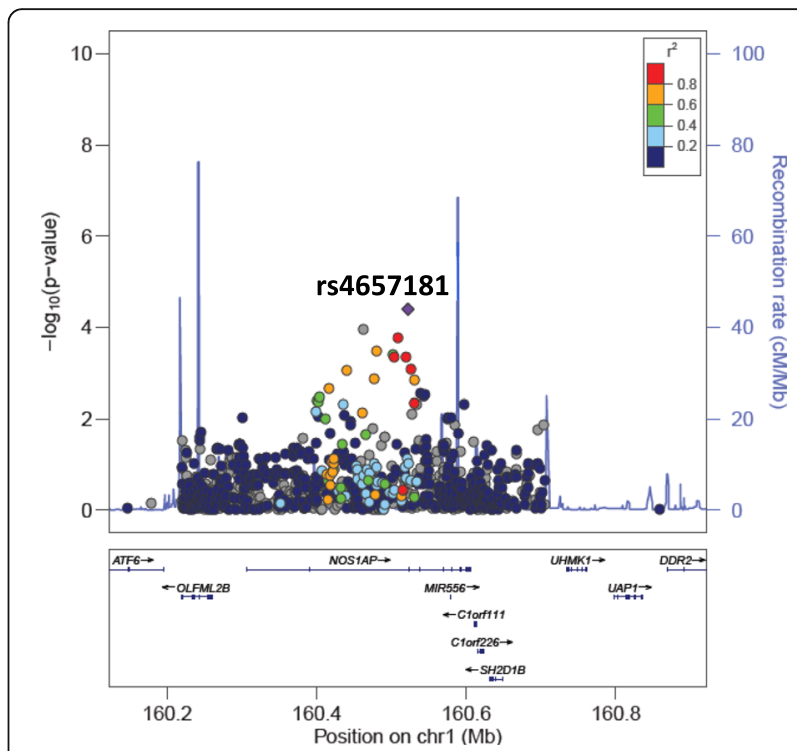

Fig. 3 LocusZoom plot for the association of rs4657181 (in NOS1AP) with SBP against a CEU LD background. rs4657181 is represented by a purple diamond. SNPs around this index SNP are coloured according to the LD between each SNP and the index SNP. SNPs with missing LD information are shown in grey. The plot shows evidence of high LD in this region in the CEU population. rs4657181 had completely missing LD information when drawn for the YRI population 

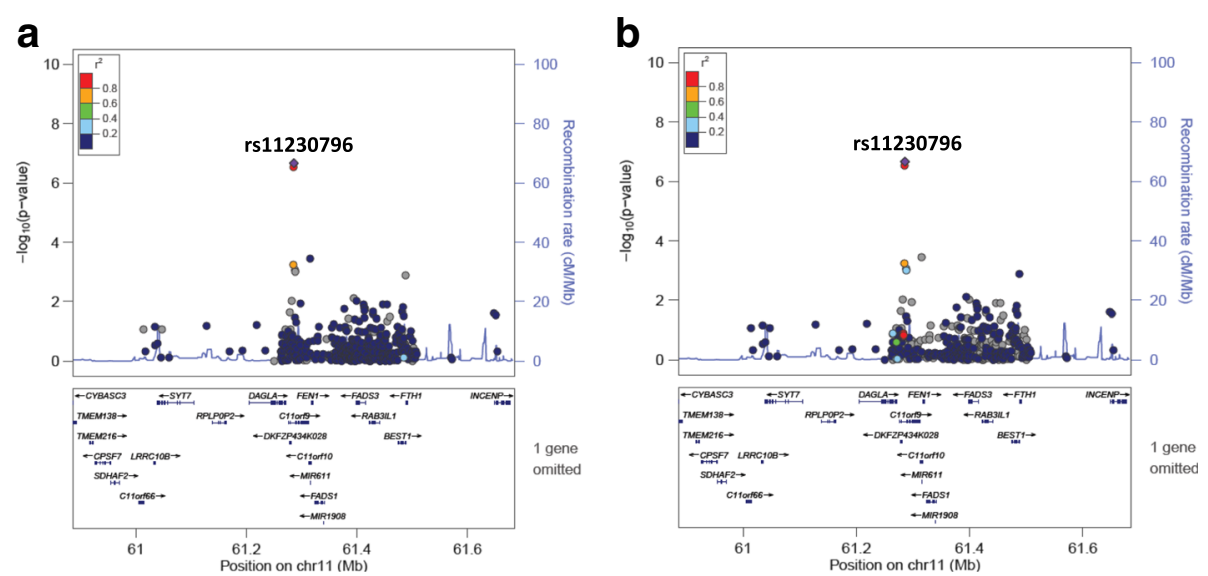

Fig. 4 LocusZoom plots for the association of rs11230796 (in MYRF) with SBP against (a) YRI and (b) CEU LD backgrounds. rs11230796 is represented by a purple diamond. SNPs around this index SNP are coloured according to the LD between each SNP and the index SNP. SNPS with missing LD information are shown in grey. MYRF is referred to by an alternative name (C110rf9) in this plot. The plot shows evidence of high LD in both the YRI and CEU populations between rs11230796 and the other SNP (rs400075) that has a strong association with SBP

It is interesting to note the marked difference in allele frequencies between this South African cohort and two other global populations (Table 2). Natural selection modulates the balance in allele frequencies across populations and variation in the frequencies of disease risk SNPs may help to explain racial disparities in disease risk [41]. It is surprising that some of the variants found to be associated with BP in this cohort are also observed at a high frequency in Europeans, yet have not been shown to be associated with BP risk in these populations. This could be explained by the differences in haplotype blocks seen in diverse populations, and the influence this may have on our ability to detect the true risk variant when using a genome-wide association study (GWAS) approach. It is now well established that association studies in Africans have the significant advantage that $\mathrm{LD}$ generally exists over a shorter genomic distance, potentially increasing the efficiency of the identification of causal variants [42].

The regional plots, generated in LocusZoom and centered around each of the lead SNPs in the identified regions of interest against YRI and CEU LD backgrounds, revealed high LD between several SNPs in NOS1AP in the CEU population (SBP) and high LD between the two associated SNPs in MYRF (SBP) and the two SNPs in POC1B (SBP) in both the YRI and CEU populations. The LD patterns in the South African population are likely to be similar and could explain the significant associations found in multiple SNPs within each region.
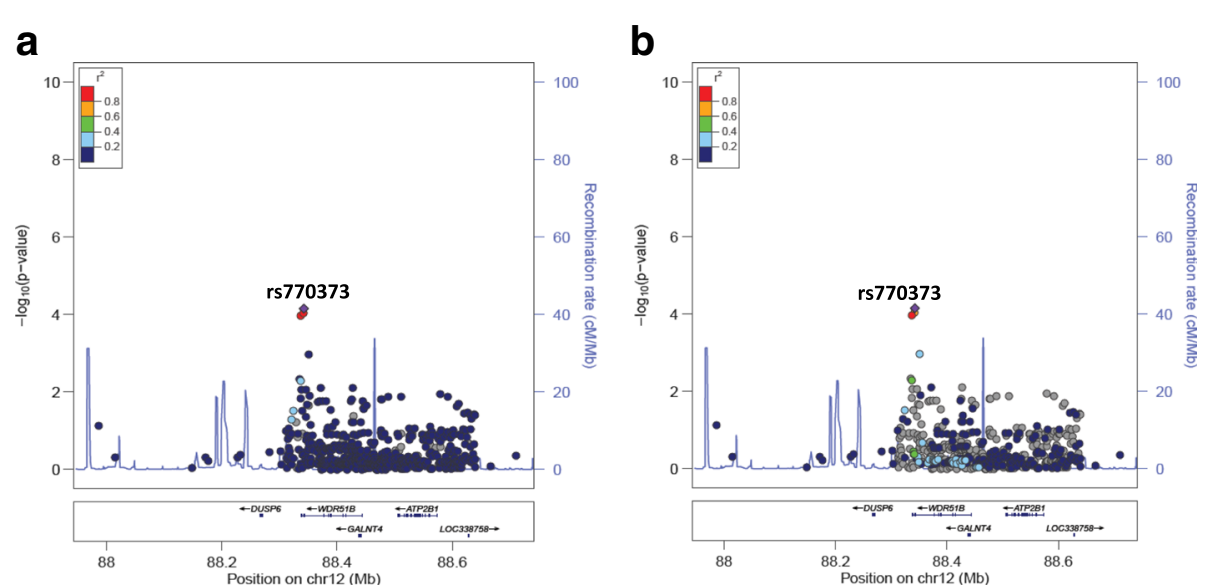

Fig. 5 LocusZoom plots for the association of rs770373 (in POC1B) with SBP against (a) YRI and (b) CEU LD backgrounds. rs 770373 is represented by a purple diamond. SNPs around this index SNP are coloured according to the LD between each SNP and the index SNP. SNPS with missing LD information are shown in grey. POC1B is referred to by an alternative name (WDR51B) in this plot. The plot shows evidence of high LD in both the YRI and CEU populations between rs770373 and the other SNP (rs770374) that has a strong association with SBP 


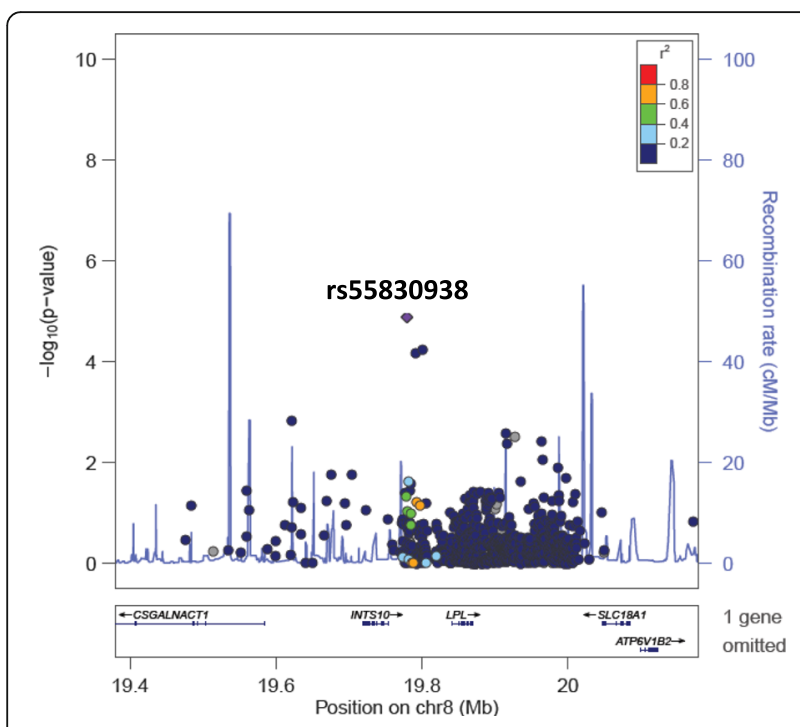

Fig. 6 LocusZoom plot for the association of rs55830938 (intergenic to INTS10 and LPL) with SBP against a YRI LD background. rs55830938 is represented by a purple diamond. SNPs around this index SNP are coloured according to the LD between each SNP and the index SNP. SNPs with missing LD information are shown in grey. rs55830938 is monomorphic in the CEU population

The Metabochip served as a good starting point in the investigation of BP genetics in black South Africans, despite the tool being developed from data on European populations. The Population Architecture using Genomics and Epidemiology (PAGE) study, whose main goal is to assess the generalizability of GWAS-identified variants across different populations, assessed the fine mapping capability of the Metabochip in African-Americans [43] and found it to be successful. Although it is known that African-Americans are genetically different to our African population, this was promising motivation for use in our black South African population. The Metabochip was developed in 2009 and several new BP/hypertension associated variants and regions have been identified since then, therefore possibly limiting the capacity to replicate in our population what has previously been found. In addition, as the Metabochip only contains variants known to be previously associated with cardiometabolic traits, the chances of identifying novel associations in Africans is reduced. A further limitation of the current study is the failure to account for the possible use of anti-hypertensive medication, specifically in the female caregivers.

\section{Conclusions}

This study has provided interesting insight into the genetics of BP in black South Africans. Studies in larger samples could enable us to identify more associated variants that have modest to small effects. The functional significance of the associations identified is unclear, though some have plausible biological explanations for their role in regulating BP. Functional and replication studies in larger African studies, as are proposed within the H3Africa Consortium [44] and more specifically the AWI-Gen study [45], will no doubt provide more insight into the genetics of BP in African populations. In addition, generalisation analysis, which has proven to be informative in a recent study on BP genetics in Hispanic/Latino Americans (another understudied population) [46], can be performed in future analyses to investigate the genetic overlap of BP between populations.

\section{Additional file}

Additional file 1: Table S1. Correlation of findings in our study with previous studies. (XLSX $13 \mathrm{~kb}$ )

\section{Abbreviations}

ARL6IP6: ADP ribosylation factor like GTPase 6 interacting protein 6; AWI-Gen: African Wits-INDEPTH Partnership for the GENomic study of body composition and cardiometabolic risk; BMl: Body mass index; BP: Blood pressure; Bt20: Birth to Twenty; CEU: Utah Residents (CEPH) with Northern and Western European Ancestry; CVD: Cardiovascular disease; DACH1: Dachshund family transcription factor 1; DBP: Diastolic blood pressure; DNA: Deoxyribonucleic acid; GWAS: Genome-wide association study; H3Africa: Human Heredity and Health in Africa; HWE: HardyWeinberg equilibrium; INTS10|LPL: Integrator complex subunit 10/lipoprotein lipase; KCNQ1: Channel, voltage gated KQT-like subfamily Q, member 1; kg: Kilograms; LD: Linkage disequilibrium; $\mathrm{m}^{2}$ : Metres squared; MAF: Minor allele frequency; MYRF: Myelin regulatory factor; NCD: Non-communicable disease; NHLS: National Health Laboratory Service; nNOS: Neuronal nitric oxide synthase; NO: Nitric oxide; NOS1AP: Nitric oxide synthase 1 (neuronal) adaptor protein; NRF: National Research Foundation; PAGE: Population Architecture using Genomics and Epidemiology; PCA: Principal component analysis; PCs: Principal components; PLEKHH2: Pleckstrin homology, MyTH4 and FERM domain containing H2; POC1B: POC1 centriolar protein B; QC: Quality control; Q-Q: Quantile-quantile; SBP: Systolic blood pressure; SCARB1: Scavenger receptor class B member 1; SNP: Single nucleotide polymorphisms; YRI: Yoruba in Ibadan, Nigeria

\section{Acknowledgements}

The authors wish to thank Richard Munthali and Scott Hazelhurst for assistance with data QC. The authors are also grateful to the participants of the Bt20 study.

\section{Funding}

This study was made possible by funding from the AWI-Gen Collaborative Centre, which is funded by the National Institutes of Health (U54HG006938) as part of the H3Africa Consortium, and the Thuthuka Programme of the South African National Research Foundation (NRF) of South Africa for the grant, Unique Grant No. 94007 \& 80702. The content is solely the responsibility of the authors and does not necessarily represent the official views of the National Institutes of Health or the NRF. LH was supported by an NRF Innovation Doctoral Scholarship for her PhD studies. Bt20 is supported by funding from the University of the Witwatersrand, South African Medical Research Council and the DST-NRF Centre of Excellence in Human Development.

\section{Availability of data and materials}

The datasets used and/or analysed during the current study are available from the corresponding author on reasonable request.

\section{Authors' contributions}

LMH performed QC, analysis and interpretation of the data and wrote the initial draft of the manuscript. VS was involved in the acquisition of genetic data, QC of the data, critically assessed the manuscript for intellectual content and approved the final version to be submitted. AC assisted with the generation of Evoker plots, offered advice/help on the QC and power calculation, critically assessed the manuscript for intellectual content and 
approved the final version to be submitted. SAN is the PI of the Bt20 cohort study, critically assessed the manuscript for intellectual content and approved the final version to be submitted. MR is the PI of the AWI-Gen study, critically assessed the manuscript for intellectual content and approved the final version to be submitted. ZL was involved in the conception and design of the study, critically assessed the manuscript for intellectual content and approved final version to be published. All authors have read and approved the final revised version of the manuscript.

\section{Ethics approval and consent to participate}

Written assent was obtained from all participants at 13 years of age in conjunction with written consent from caregivers prior to blood sample collection. Written consent was obtained from participants when they were over 18 years of age. Ethical clearance was obtained from the University of the Witwatersrand Human Research Ethics Committee (Medical) for collection of DNA samples and phenotype data from this cohort (M010556). Further clearance was obtained for use of these samples to identify genetic risks associated with blood pressure (M1411116) in a black South African population.

\section{Consent for publication}

Not applicable

\section{Competing interests}

The authors declare that they have no competing interests.

\section{Publisher's Note}

Springer Nature remains neutral with regard to jurisdictional claims in published maps and institutional affiliations.

\begin{abstract}
Author details
'School of Molecular \& Cell Biology, Faculty of Science, University of the Witwatersrand, Johannesburg, South Africa. ${ }^{2}$ Sydney Brenner Institute for Molecular Bioscience, Faculty of Health Sciences, University of the Witwatersrand, Johannesburg, South Africa. ${ }^{3}$ Division of Human Genetics, School of Pathology, Faculty of Health Sciences, National Health Laboratory Service \& University of the Witwatersrand, Johannesburg, South Africa. ${ }^{4} \mathrm{MRC} /$ Wits Developmental Pathways for Health Research Unit, Department of Paediatrics, School of Clinical Medicine, Faculty of Health Sciences, University of the Witwatersrand, Johannesburg, South Africa.
\end{abstract}

Received: 20 September 2017 Accepted: 3 January 2018

Published online: 17 January 2018

\section{References}

1. World Health Organization. Global status report on noncommunicable diseases. 2014:2014:176.

2. Munroe PB, Barnes MR, Caulfield MJ. Advances in blood pressure genomics. Circ Res. 2013;112:1365-79.

3. Doris PA. Hypertension genetics, single nucleotide polymorphisms, and the common disease:common variant hypothesis. Hypertension. 2002:39:323-31.

4. Franceschini N, Le T. Genetics of hypertension: discoveries from the bench to human populations. Am. J. Physiol. - Ren Physiol. 2013;306:F1-11.

5. Popejoy A, Fullerton S. Genomics is failing on diversity. Nature. 2016:538:161-4

6. Richter L, Norris S, Pettifor J, Yach D, Cameron N. Cohort profile: Mandela's children: the 1990 Birth to Twenty study in South Africa. Int J Epidemiol. 2007;36:504-11.

7. Kagura J, Adair LS, Musa MG, Pettifor JM, Norris SA. Blood pressure tracking in urban black south African children: Birth to Twenty cohort. BMC Pediatr. 2015:15:1-7.

8. Miller SA, Dykes DD, Polesky HFA. Simple salting out procedure for extracting DNA from human nucleated cells. Nucleic Acids Res. 1988;16:1215

9. Voight BF, Kang HM, Ding J, Palmer CD, Sidore C, Chines PS, et al. The metabochip, a custom genotyping array for genetic studies of metabolic, cardiovascular, and anthropometric traits. PLoS Genet. 2012/08/10. 2012:8:e1002793.

10. Purcell S, Neale B, Todd-Brown K, Thomas L, Ferreira MA, Bender D, et al. PLINK: a tool set for whole-genome association and population-based linkage analyses. Am J Hum Genet. 2007;81:559-75.
11. Purcell S, Chang C. PLINK 1.9 [Internet]. 2014. Available from: https://www. cog-genomics.org/plink2

12. Patterson N, Price AL, Reich D. Population structure and eigenanalysis. PLoS Genet. 2006:2:e190.

13. Buchmann R, Hazelhurst S. Genesis manual [internet]. 2014. Available from: http://www.bioinf.wits.ac.za/software/genesis/Genesis.pdf

14. Anderson CA, Pettersson FH, Clarke GM, Cardon LR, Morris AP, Zondervan $\mathrm{KT}$. Data quality control in genetic case-control association studies. Nat Protoc. 2010;5:1564-73.

15. Morris JA, Randall JC, Maller JB, Barrett JC. Evoker: a visualization tool for genotype intensity data. Bioinformatics. 2010;26:1786-7.

16. Zhou X, Stephens M. Genome-wide efficient mixed-model analysis for association studies. Nat Genet. 2012;44:821-4.

17. R Development Core Team. R: A language and environment for statistical computing. R Found. Stat. Comput. Vienna, Austria; 2009.

18. Arking DE, Pfeufer A, Post W, Kao WHL, Newton-Cheh C, Ikeda M, et al. A common genetic variant in the NOS1 regulator NOS1AP modulates cardiac repolarization. Nat Genet. 2006:38:644-51.

19. Faruque MU, Chen G, Doumatey A, Huang H, Zhou J, Dunston GM, et al. Association of ATP1B1, RGS5 and SELE polymorphisms with hypertension and blood pressure in African-Americans. J Hypertens. 2011;29:1906-12.

20. Melikian N, Seddon MD, Casadei B, Chowienczyk PJ, Shah AM. Neuronal nitric oxide synthase and human vascular regulation. Trends Cardiovasc Med. 2009:256-62

21. Hermann M, Flammer A, Lüscher TF. Nitric oxide in hypertension. J Clin Hypertens. 2006:8:17-29.

22. Förstermann $U$, Sessa WC. Nitric oxide synthases: regulation and function. Eur Heart J. 2012:829-37.

23. C-J L, Hao G, Nikiforova N, Larsen HE, Liu K, Crabtree MJ, et al. CAPON modulates neuronal calcium handling and cardiac sympathetic neurotransmission during dysautonomia in hypertension. Hypertension. 2015;65:1288-97.

24. Lemaitre RN, Tanaka T, Tang W, Manichaikul A, Foy M, Kabagambe EK, et al. Genetic loci associated with plasma phospholipid n-3 fatty acids: a metaanalysis of genome-wide association studies from the CHARGE consortium. PLoS Genet. 2011;7:e1002193.

25. Shin S-Y, Fauman EB, Petersen A-K, Krumsiek J, Santos R, Huang J, et al. An atlas of genetic influences on human blood metabolites. Nat Genet. 2014:46:543-50

26. Mozaffarian D, Kabagambe EK, Johnson CO, Lemaitre RN, Manichaikul A, Sun Q, et al. Genetic loci associated with circulating phospholipid trans fatty acids: a meta-analysis of genome-wide association studies from the CHARGE consortium. Am J Clin Nutr. 2015;101:398-406.

27. Tintle NL, Pottala JV, Lacey S, Ramachandran V, Westra J, Rogers A, et al. A genome-wide association study of fourteen red blood cell fatty acids in the Framingham heart study. Prostaglandins. Leukot Essent Fat Acids. 2015;94:65-72.

28. Leslie R, O'Donnell CJ, Johnson ADGRASP. Analysis of genotype-phenotype results from 1390 genome-wide association studies and corresponding open access database. Bioinformatics. 2014;30

29. Staley JR, Blackshaw J, Kamat MA, Ellis S, Surendran P, Sun BB, et al. PhenoScanner: a database of human genotype-phenotype associations. Bioinformatics. 2016;32:3207-9.

30. Ehret GB, Munroe PB, Rice KM, Bochud M, Johnson AD, Chasman DI, et al. Genetic variants in novel pathways influence blood pressure and cardiovascular disease risk. Nature. 2011/09/13. 2011:478:103-109.

31. Suchindran S, Rivedal D, Guyton JR, Milledge T, Gao X, Benjamin A, et al. Genome-wide association study of Lp-PLA2 activity and mass in the framingham heart study. PLoS Genet. 2010;6

32. Grallert H, Dupuis J, Bis JC, Dehghan A, Barbalic M, Baumert J, et al. Eight genetic loci associated with variation in lipoprotein-associated phospholipase A2 mass and activity and coronary heart disease: metaanalysis of genome-wide association studies from five community-based studies. Eur Heart J. 2012;33:238-51.

33. Kettunen J, Tukiainen $T$, Sarin A-P, Ortega-Alonso A, Tikkanen $E$, Lyytikäinen $L-P$, et al. Genome-wide association study identifies multiple loci influencing human serum metabolite levels. Nat Genet. 2012:44:269-76.

34. Hiura Y, Tabara Y, Kokubo Y, Okamura T, Miki T, Tomoike H, et al. A genome-wide association study of hypertension-related phenotypes in a Japanese population. Circ J. 2010;74:2353-9. 
35. Zhu X, Young JH, Fox E, Keating BJ, Franceschini N, Kang S, et al. Combined admixture mapping and association analysis identifies a novel blood pressure genetic locus on 5p13: contributions from the CARe consortium. Hum. Mol. Genet. 2011/03/23. 2011:20:2285-2295.

36. Fox ER, Young JH, Li Y, Dreisbach AW, Keating BJ, Musani SK, et al. Association of genetic variation with systolic and diastolic blood pressure among African Americans: the candidate Gene Association resource study. Hum Mol Genet. 2011;20:2273-84.

37. Tomaszewski M, Debiec R, Braund PS, Nelson CP, Hardwick R, Christofidou P, et al. Genetic architecture of ambulatory blood pressure in the general population: insights from cardiovascular gene-centric array. Hypertension. 2010;56:1069-76.

38. Yang HC, Liang YJ, Chen JW, Chiang KM, Chung CM, Ho HY, et al. Identification of IGF1, SLC4A4, WWOX, and SFMBT1 as hypertension susceptibility genes in Han Chinese with a genome-wide gene-based association study. PLoS One. 2012;7

39. Burton PR, Clayton DG, Cardon LR, Craddock N, Deloukas P, Duncanson A, et al. Genome-wide association study of 14,000 cases of seven common diseases and 3,000 shared controls. Nature. 2007;447:661-78.

40. Liang J, Le TH, Edwards DRV, Tayo BO, Gaulton KJ, Smith JA, et al. Singletrait and multi-trait genome-wide association analyses identify novel loci for blood pressure in African-ancestry populations. PLoS Genet. 2017;13:e1006728

41. Mattei J, Parnell L, Lai C, Garcia-Bailo B, Adiconis X, Shen J, et al. Disparities in allele frequencies and population differentiation for 101 diseaseassociated single nucleotide polymorphisms between Puerto Ricans and non-Hispanic whites. BMC Genet. 2009;10

42. Teo Y-Y, Small KS, Kwiatkowski DP. Methodological challenges of genomewide association analysis in Africa. Nat Rev Genet. 2010;11:149-60.

43. Buyske S, Wu Y, Carty CL, Cheng I, Assimes TL, Dumitrescu L, et al. Evaluation of the metabochip genotyping array in African Americans and implications for fine mapping of GWAS-identified loci: the PAGE study. PLoS One. 2012;7:e35651.

44. H3Africa Consortium. Enabling the genomic revolution in Africa. Science (80-. ). 2014:344:1346-8.

45. Ramsay M, Crowther N, Tambo E, Agongo G, Baloyi V, Dikotope S, et al. H3Africa AWI-gen collaborative Centre: a resource to study the interplay between genomic and environmental risk factors for cardiometabolic diseases in four sub-Saharan African countries. Glob. Heal. Epidemiol. Genomics. 2016;1:1-13.

46. Sofer T, Wong Q, Hartwig F, Taylor K, Warren H, Evangelou E, et al. Genomewide association study of blood pressure traits by Hispanic/Latino background: the hispanic community health study/study of Latinos. Sci. Rep. 2107;7

\section{Submit your next manuscript to BioMed Central and we will help you at every step:}

- We accept pre-submission inquiries

- Our selector tool helps you to find the most relevant journal

- We provide round the clock customer support

- Convenient online submission

- Thorough peer review

- Inclusion in PubMed and all major indexing services

- Maximum visibility for your research

Submit your manuscript at www.biomedcentral.com/submit

CBiomed Central 